\title{
Calidad de los servicios logísticos
}

Quality of logistics services

Qualidade de serviços de logística

DOI: http://dx.doi.org/10.23913/ricea.v6i11.89

\author{
Sandoval Chávez Rebeca \\ Universidad Politécnica Gómez Palacio, México \\ rsandoval@upgop.edu.mx \\ Juan Diego Hinojosa Escajeda \\ Instituto Tecnológico de la Laguna, México \\ jd_hinojosa1@yahoo.com.mx \\ María Guadalupe Sandoval Chávez \\ Instituto Tecnológico de la Laguna, México \\ sandoval_li@yahoo.com.mx
}

\section{Resumen}

Esta investigación es la segunda parte del artículo "Prestadores de Servicios de apoyo a la exportación en la Comarca Lagunera”, publicado en la Revista Iberoamericana de Contaduría, Economía y Administración, vol.5, núm.10, donde se hace un recuento de los prestadores del servicio logístico.

El éxito, la reducción de costes y la satisfacción de los clientes de una organización dependen de un sistema logístico bien gestionado, integrado y flexible, controlado en tiempo real y donde fluye la información de manera eficiente (IDE-CESEM, 2017).

El objetivo de esta investigación es medir la calidad de los servicios logísticos en la Comarca Lagunera de acuerdo a tres dimensiones: conformidad, desempeño y confiabilidad, las cuales a su vez se desglosan en tres áreas: transporte, oficinas gubernamentales e instituciones financieras.

Los principales resultados fueron: $35 \%$ de las empresas encuestadas realiza tanto exportaciones como importaciones, $36 \%$ solamente exporta y $29 \%$ importa; asimismo, $54 \%$ realiza 
operaciones de comercio exterior con una frecuencia semanal y el restante $46 \%$ de manera mensual.

Los empresarios señalan que las áreas donde más les afecta la falta de confiabilidad y el desempeño de sus prestadores de servicio son el transporte y la distribución de los productos. Su dificultad para manejar niveles adecuados en el almacén proviene de la incertidumbre con respecto al transporte y los tiempos de recepción de la materia prima.

En términos generales, la oportunidad de mejorar la calidad de los servicios asciende a $57 \%$, mientras que las áreas de transporte aéreo y servicios financieros alcanzaron la calificación más baja. Por otro lado, $37 \%$ de los exportadores e importadores considera que el precio que pagan por el transporte es elevado dada la contraprestación que reciben.

Otro resultado importante es que $56 \%$ de las empresas encuestadas afirma que no conoce fuentes de financiamiento para la exportación y/o importación, dato confirmado con el ITEM 14 (¿Conoce los servicios que ofrece Bancomext, Pro México?), el cual arrojó que 4 de cada 10 empresas no conocen la gama de servicios que ofrecen estas instituciones.

Palabras clave: calidad, exportación, importación, logística, servicio.

\section{Abstract}

This research is the second part of the article entitled "Providers of Export Support Services in the Comarca Lagunera" published in the Ibero-American Journal of Accounting, Economy and Administration, vol. 5, no. 10, where a count of the logistics service providers is made.

The success of an organization, the reduction of costs and the satisfaction of its clients' needs depend on a well managed, integrated and flexible logistics system controlled in real time and in which information flows efficiently (IDE-CESEM, 2017).

This is why the present research aims to measure the quality of logistics services in the Comarca Lagunera according to three dimensions: compliance, performance and reliability, which in turn are broken down into three areas of expertise: Transportation, government offices and financial institutions.

Among the main obtained results the next ones are pointed up: 
$35 \%$ of the companies surveyed carry out both exports and imports, $36 \%$ only exports and $29 \%$ imports.

$54 \%$ carry out foreign trade operations with a weekly frequency and the remaining $46 \%$ monthly.

Entrepreneurs point out that the areas where the lack of reliability and performance of their service providers impact are the transportation and distribution of products, followed by the difficulty of managing the appropriate levels of warehouse by the uncertainty the opportunity of transportion generates and not less important the reception times of raw material.

In general terms, the area of opportunity to improve the quality of services reports an average of 57\%, with air transport and financial services having the lowest rating. 37\% of exporters and importers consider that the price they pay for transportation is high in function of the counterpayment they receive.

Another important find is that $56 \%$ of the companies surveyed say not to know sources of financing for export and / or import, which is confirmed by ITEM 14 (Do you know the services offered by Bancomext, Pro Mexico?) showing that 4 of each 10 do not know the range of services offered by these institutions.

Key words: quality, export, import, logistics, service.

\section{Resumo}

Esta pesquisa é a segunda parte do "apoio Fornecedores de Serviços exportação na Região Laguna" do artigo, publicado na Revista Iberoamericana de Contabilidade, Economia e Gestão, vol.5, no.10, onde a contagem é feita prestadores de serviços de logística.

Sucesso, custos reduc $\neg$ ción e satisfação do cliente em uma organização Depend-den gestiona $\neg$ do um sistema de logística flexíveis bem integrada e controlada em tempo real e onde a informação de forma eficiente (IDE-CESEM flui , 2017).

O objetivo desta pesquisa é medir a qualidade dos serviços de logística na região de Laguna de acordo com três dimensões: conformidade, desempenho e confiabilidade, que por sua vez é dividido em três áreas: transporte, escritórios do governo e instituições financeiras. 
Os principais resultados foram: $35 \%$ das empresas pesquisadas realizada exportações e importações, exportações apenas $36 \%$ e $29 \%$ de matéria; também $54 \%$ faz comércio exterior numa base semanal e os restantes $46 \%$, numa base mensal.

Empresários afirmam que as áreas mais afetadas pela falta de confiabilidade e desempenho de seus prestadores de serviços são o transporte e distribuição de produtos. Dificuldade em gerir os seus níveis adequados na loja vem da incerteza quanto ao transporte e tempo de recebimento da matéria-prima.

De um modo geral, a oportunidade de melhorar a qualidade dos serviços é de $57 \%$, enquanto que as áreas de transporte aéreo e aos serviços financeiros alcançou a classificação mais baixa. Por outro lado, 37\% dos exportadores e importadores considera que o preço que pagam para o transporte é elevado dada a consideração que recebem.

Outro resultado importante é que 56\% das empresas pesquisadas afirmaram que há fontes conhecidas de financiamento para exportação e / ou importação de dados confirmados com o item 14 (Você conhece os serviços que Bancomext oferece, Pro México?), Que mostrou que 4 em cada 10 empresas não sabem a gama de serviços oferecidos por estas instituições.

Palavras-chave: qualidade, exportação, importação, logística, serviço.

Fecha recepción: Agosto 2016 Fecha aceptación: Diciembre 2016

\section{Introducción}

Los avances tecnológicos actuales en el mercado facilitan la productividad de los procesos en las empresas y sectores comerciales. Existe una amplia diversidad de servicios que se ofrecen a las empresas que desean exportar o importar, por lo que es bueno conocer el grado de satisfacción de los usuarios de servicios logísticos y de apoyo a la exportación e importación.

Hasta junio del 2016, el valor de las exportaciones de mercancías a nivel nacional fue de 31942 millones de dólares, es decir, la suma de 30326 millones de dólares de exportaciones no petroleras y 1617 millones de dólares de exportaciones petroleras (INEGI, 2017). De acuerdo a este dato, $94.94 \%$ de las exportaciones las realiza el sector empresarial (es decir, $3.51 \%$ sector agropecuario, $30.25 \%$ manufactureras automotrices y $59.94 \%$ manufactureras no automotrices), 
de ahí la importancia de contar con la infraestructura y los servicios de calidad que apoyen a la exportación.

Por otra parte, $80 \%$ de la producción agrícola lagunera que se obtiene de agricultura protegida es de gran calidad, así que se puede vender en el mercado internacional (Milenio, 2014). Además, según datos de la Dirección de Fomento Económico, 30 \% de las empresas de la Región Lagunera exporta sus productos (Cía. Editora de la Laguna S.A. de C.V, 2017).

Los siguientes autores definen "servicio" de las siguientes maneras:

Para Fisher y Navarro (1994), el servicio es "un tipo de bien económico que constituye lo que denomina el sector terciario, todo el que trabaja y no produce bienes se supone que produce servicios". Por su parte, Cantú (2011) menciona que "un servicio es la actividad o conjunto de actividades de naturaleza casi siempre intangible que se realiza mediante la interacción entre el cliente y el empleado y/o las instalaciones físicas de servicio, a fin de satisfacer un deseo o una necesidad de usuario". Kotler (2007) lo enuncia como "cualquier actividad o beneficio que una parte ofrece a otra; son esencialmente intangibles y no dan lugar a la propiedad de ninguna cosa. Su producción puede estar vinculada o no con un producto físico".

La calidad de los servicios es uno de los factores que más influyen en la expansión y triunfo de las empresas frente a la competencia (Gestión de los servicios socio-sanitarios, 2015).

Un servicio de calidad ofrece garantía a todos, tanto empresas como consumidores (Principios de Gestión de Calidad, 2014):

El servicio de calidad es algo que nunca termina, pues siempre debe tender a mejorar. Es necesario aplicar estándares de calidad muy altos. El servicio debe ser lo suficientemente bueno para diferenciar a una empresa de las demás.

La calidad de los servicios se consigue con un personal implicado, consciente de que un error es un exceso, y por ende algo que se debe evitar. Para ello es indispensable contar con un buen ambiente laboral, con personal con buena experiencia, con una actitud de entrega a la perfección y, sobre todo, con una dirección que lidere el proceso. 
Los beneficios de la calidad (Principios de Gestión de Calidad, 2014) son:

- A corto plazo: mayor beneficio para la empresa debido a una mayor venta e incremento de su precio.

- A largo plazo: la empresa empieza a crecer.

\section{¿Qué son los servicios logísticos?}

La logística es una red de medios, métodos e infraestructuras encargadas de garantizar el almacenamiento, transporte y la entrega de bienes y servicios. Está íntimamente relacionada con los medios de transportes, ya que es el medio de envío de productos y mercancías hacia otra zona geográfica (Paul R. Murphy Jr, 2015).

Un operador logístico es una empresa especializada que una compañía subcontrata para que actúe como su departamento logístico. Hay diversos tipos de operador logístico (Paul R. Murphy Jr, 2015):

- 1PL: First Party Logistics. Son aquellos operadores que se ocupan únicamente del transporte de las mercancías; empresas de transporte que desplazan sus unidades hasta el almacén de la compañía que ha contratado sus servicios para distribuir sus productos.

- 2PL: Second Party Logistics. Se ocupa del transporte y del almacenamiento de la mercancía. Gestiona la distribución y el almacenaje, por lo que cuenta con unidades de transporte y con uno o varios almacenes.

- 3PL: Third Party Logistics. Estos operadores logísticos gestionan y verifican todo el proceso logístico para optimizar al máximo la producción y distribución de la empresa que contrata sus servicios.

- 4PL: Fourth Party Logistics. El operador se encarga de optimizar tanto la administración logística como la cadena de suministros. 


\section{¿Qué es exportar?}

Es cualquier bien o servicio enviado fuera del territorio nacional. Gracias a esto, las microempresas tienen la oportunidad de expandirse en su propio continente e incluso en otros. El crecimiento de una empresa significa mayor solicitud de personal y mano de obra, lo que crea muchas oportunidades de desempeño, así como mayores inversiones y beneficios económicos (Aduanas, 2010).

Finalidad de la exportación: asegurar el continuo crecimiento de la empresa que vende en otros países. La actividad exportadora es una venta y en la economía de mercado su principal objetivo es lograr beneficios para la empresa que realiza ventas de exportación. Aumentar las utilidades es la motivación fundamental de toda exportación (Mercado Hernández, 2012).

La empresa diversifica riesgos, experimenta en el mercado mundial las variaciones del gusto de los consumidores y las novedades de los productos de la competencia, aprendiendo en la gran escuela del comercio internacional los sistemas y formas más recientes para vender y ampliar ventas, mejorar sus productos y obtener una serie de ventajas que van desde posibles fuentes de financiamiento externo hasta una mejor imagen en el propio mercado nacional (Mercado Hernández, 2012).

\section{¿Qué es importar?}

Es la compra de un bien o servicio que proviene de un país extranjero. Toda importación permite comprar productos que no son elaborados en el país o de los cuales existe escasez; pueden ser más baratos o de mejor calidad (Aduanas, 2010).

\section{¿Qué es la banca?}

Son las entidades o instituciones que dentro de una economía determinada prestan el servicio de banco. Se utiliza este término como sinónimo o equivalente del concepto de banco, que es la organización cuya función es tomar los recursos o dinero de las personas, empresas u otro tipo de organizaciones y con ellos otorgar créditos a aquellos que los soliciten; es decir, realizan dos actividades fundamentales: captación y colocación (Banco de México, 2017).

Bancos de primer piso

Instituciones legalmente autorizadas para realizar operaciones de ahorro, financiación, hipotecarias y capitalización. Tienen relación directa con los clientes (Jhon Fredy Rave, 2017). 
Bancos de segundo piso

Fondos creados por el gobierno para el desarrollo de algún sector de la economía en particular. Sus características principales son que no operan directamente con particulares y que obtienen los créditos a través de instituciones privadas de crédito. De ahí el origen del nombre "bancos de segundo piso", es decir, no hay trato o acceso directo a particulares (Jhon Fredy Rave, 2017).

\section{Agente aduanal}

Es la persona física autorizada por la Secretaría de Hacienda y Crédito Público mediante una patente para promover por cuenta ajena el despacho de las mercancías en los diferentes regímenes previstos por esta ley. No es un funcionario público, sino un integrante de la iniciativa privada que coadyuva con la Secretaría de Hacienda para el control de la entrada y salida de mercancías, el correcto pago de contribuciones y cuotas compensatorias (Confederación de Asociaciones de Agentes Aduanales de la República Mexicana, 2017).

El agente aduanal es uno de los principales aliados estratégicos de las empresas mexicanas ya que se les proporciona un servicio de "outsourcing aduanero", que permite a las empresas reducir costos importantes de operación y control de sus operaciones y al fisco federal coadyuvar con la fiscalización y recaudación de las contribuciones al comercio exterior. Como dato interesante está que los agentes aduanales recaudan $44 \%$ del IVA nacional. Además, el agente aduanal (Confederación de Asociaciones de Agentes Aduanales de la República Mexicana, 2017) verifica lo siguiente:

- Permiso previo de importación ante la Secretaría de Economía.

- Certificados de origen.

- Tratados y acuerdos comerciales que México haya suscrito con otros países.

- Normas Oficiales Mexicanas.

- Autorizaciones de salud.

- Permisos de sanidad o fitosanitarios.

- Autorizaciones de protección ambiental.

- Etiquetados de información comercial.

- Marcados de país de origen.

- Inspecciones de autoridades diversas. 
Según el Banco de México, el total de las mercancías que se importan y exportan anualmente al país equivalen al $67 \%$ del Producto Interno Bruto de México, lo cual explica la importancia de la figura del agente aduanal.

\section{Modelos de medición de la calidad del servicio al cliente}

\section{La escuela nórdica}

Este modelo, también conocido como de imagen corporativa, plantea que la calidad percibida por los clientes es la integración de la calidad técnica (lo que se da) y la calidad funcional (la manera como se da), las cuales se relacionan con la imagen corporativa. La imagen es un elemento básico para medir la calidad percibida, por tanto, el cliente está influido por el resultado del servicio, pero también por la forma en que lo recibe y la imagen corporativa. Todo ello estudia transversalmente las diferencias entre el servicio esperado y la percepción del servicio (Grönroos, 1994).

Una buena evaluación de la calidad percibida se obtiene cuando la calidad experimentada cumple con las expectativas del cliente, es decir, lo satisface. De igual forma, el exceso de expectativas genera problemas en la evaluación de la calidad (Grönroos, 1994).

Expectativas poco realistas contrastadas con calidad experimentada como buena pueden desembocar en una calidad total percibida como baja. Las expectativas o calidad esperada, según Grönroos, están en función de factores como la comunicación de marketing, recomendaciones (comunicación boca-oído), imagen corporativa/local y las necesidades del cliente.

Componentes (Grönroos, 1994):

- La calidad técnica se enfoca en un servicio técnicamente correcto que conduce hacia un resultado aceptable. Se preocupa de todo lo concerniente al soporte físico, los medios materiales y la organización interna (la manera como el consumidor recibe el servicio).

- La calidad funcional es la forma en que los consumidores perciben la empresa; es la imagen corporativa de la empresa. Es la percepción de la calidad técnica y funcional de los servicios que presta una organización y, por tanto, influye en la percepción global del servicio. 


\section{Escuela americana}

Afirma que son cinco las dimensiones que determinan la calidad del servicio (A. Parasuraman, 2017):

- Confianza o empatía: muestra de interés y nivel de atención individualizada que ofrecen las empresas a sus clientes (agrupa los anteriores criterios de accesibilidad, comunicación y comprensión del usuario).

- Fiabilidad: habilidad para ejecutar el servicio prometido de forma fiable y cuidadosa.

- Responsabilidad: seguridad, conocimiento y atención de los empleados y su habilidad para inspirar credibilidad y confianza (agrupa las anteriores dimensiones de profesionalidad, cortesía, credibilidad y seguridad).

- Capacidad de respuesta: disposición para ayudar a los clientes y para prestarles un servicio rápido.

- Tangibilidad: apariencia de las instalaciones físicas, equipos, personal y materiales de comunicación.

Para evaluar la calidad percibida se plantean estas dimensiones generales, que definen que dicha percepción es consecuencia de la diferencia para el consumidor entre lo esperado y lo percibido.

Parasuraman, Zeithaml y Berry (2017) definen vacío o gap como una serie de discrepancias o deficiencias existentes respecto a las percepciones de la calidad de servicio de los ejecutivos y las tareas asociadas con el servicio que se presta a los consumidores. Estas deficiencias son los factores que afectan la imposibilidad de ofrecer un servicio que sea percibido por los clientes como de alta calidad.

\section{Método}

La investigación se desarrolló en la Comarca Lagunera, zona localizada en el Bolsón de Mapimí y las Lagunas de Mayrán- Viesca. Está conformada por 6 municipios del estado de Coahuila y 14 del estado de Durango. Dentro de ella se encuentra la novena zona metropolitana más poblada de México, considerada una de las zonas conurbadas más jóvenes y de mayor crecimiento en México (INEGI, 2017). 
El tipo de investigación es descriptivo. El estudio comprende tres dimensiones: conformidad, desempeño, confiabilidad. Las 3 dimensiones se desglosaron en 3 áreas de dominio (transporte, oficinas gubernamentales, instituciones financieras). Se desarrollaron instrumentos denominados "Calidad de los servicios logísticos en la Comarca Lagunera", validado con Alpha Cronbach con un resultado de [a=0.897276]. La determinación de la muestra fue: 104 encuestas para 20 ítems (Cervantes, 2005), con el tamaño de la muestra en relación al número de ítems: regla: entre 5 y 20 encuestas por ítem. El tamaño de la muestra fue entre 100 y 400 encuestas.

Los datos utilizados fueron: exportadores e importadores de la Comarca Lagunera asentados en el directorio de PROSEC, de la Secretaría de Economía. Se capturó el instrumento aplicado al programa estadístico, y se elaboraron la estadística y el análisis de resultados.

\section{Resultados}

Paradigma cualitativo

De acuerdo al objetivo de la investigación se seleccionaron 3 dimensiones (conformidad, desempeño, confiabilidad), las cuales se desglosaron en las siguientes 3 áreas de dominio:

Medios de transporte: un transporte eficiente y la compresión de sus reglas de operación es un potencial de mejora de la competitividad en las empresas.

Oficinas gubernamentales: es de suma importancia que tanto exportadores como importadores conozcan el cúmulo de requisitos regulatorios que implica hacer un intercambio comercial, que va desde la fracción arancelaria hasta las regulaciones fitosanitarias que hay que acatar. Para cada tipo de trámite existe alguna instancia responsable de cuidar que esto se cumpla, así que la calidad del servicio que brindan es fundamental para llevar a buen término una operación de comercio internacional.

Instituciones financieras: los servicios que ofrecen van desde la expedición de una carta de crédito, el factoraje y el crédito para capital de trabajo, un apoyo fundamental al comercio internacional. También es importante destacar el papel que juega la banca de desarrollo, cuya función principal es proveer de servicio financiero a los sectores prioritarios para el desarrollo 
económico del país, motivo por el cual la presente investigación lo incluye en la evaluación de los servicios.

\section{Cuantitativo}

Las respuestas al cuestionario "Calidad de los servicios logísticos en la Comarca Lagunera" fueron capturadas y posteriormente analizadas mediante software computacional de estadística, lo que permitió evidenciar la captura y a su vez entregar la información estadística correspondiente.

De acuerdo a dichos datos:

- $\quad 35 \%$ de las empresas encuestadas realiza tanto exportaciones como importaciones, $36 \%$ solo exporta y $29 \%$ importa.

- $\quad 98 \%$ de los encuestados está familiarizado con el término logística e incluso lo maneja al interior de sus organizaciones.

- $\quad 54 \%$ realiza operaciones de comercio exterior con una frecuencia semanal y el restante $46 \%$ de manera mensual.

A la pregunta específica formulada a los empresarios sobre si identificaban el tipo de problemas que les causaba la calidad de los servicios logísticos, solo $1 \%$ respondió que no, mientras que el restante 99 \% sí tiene claro cuáles son las áreas en las que les impacta la falta de confiabilidad y desempeño de sus prestadores de servicio. La gráfica 1 muestra el predominio del transporte y la distribución de productos, seguidos de la dificultad de manejar niveles adecuados de almacén debida a la incertidumbre sobre el transporte y los tiempos de recepción de la materia prima.

Por otro lado, $5 \%$ de los encuestados utiliza transporte multimodal, siendo el terrestre el que más predominó (gráfica 2).

La gráfica 3 muestra que el área de oportunidad para mejorar la calidad de los servicios reporta un promedio general de $57 \%$, siendo el transporte aéreo y los servicios financieros los que obtuvieron la calificación más baja. Solo el servicio financiero, el transporte terrestre y los servicios de las agencias aduanales alcanzaron una calificación de excelencia, aunque un porcentaje muy bajo $(1 \%)$.

Por otra parte, $86 \%$ de los encuestados considera apropiadas las rutas de los ferrocarriles, mientras que $14 \%$ dice que hay que mejorarlas, y $37 \%$ de los exportadores e importadores 
considera que el precio que pagan por el transporte es elevado tomando en cuenta la contraprestación que reciben.

Dos de cada diez empresas contestaron que los costos de exportación son en general altos, y dos de cada diez empresarios califican las tasas de interés como muy elevadas; además, $56 \%$ de las empresas encuestadas dice no conocer fuentes de financiamiento para la exportación y/o importación, dato que se confirma con el ITEM 14 (¿Conoce los servicios que ofrece Bancomext, Pro México?), el cual arrojó que 4 de cada 10 no conocen la gama de servicios que ofrecen estas instituciones.

La instancia que obtuvo la peor calificación en la oportunidad de los servicios que brinda fueron las oficinas de gobierno con $30 \%$ (gráfica 4). Y las mejor calificadas fueron las agencias aduanales, con la observación de que deberían ampliar su servicio las 24 horas en el despacho de mercancías.

Por otra parte, 8 de cada 10 empresas cuentan con convenios o enlaces fronterizos.

\section{Conclusión}

Las razones por las cuales una empresa decide iniciarse en el proceso de exportación e importación son muy variadas: diversificación de mercados, adquirir experiencia y alianzas estratégicas, búsqueda de ventas en mayor volumen, hacer economía de escala, ganar competitividad, intercambio tecnológico, disminuir riesgo de un solo mercado, búsqueda de mayor rentabilidad, entre otras (PROCOLOMBIA, 2017).

Los actores principales de este proceso son los prestadores de servicios logísticos, ya que deben llevar a buen término una operación de comercio internacional.

La calidad de los prestadores de servicios logísticos se adiciona a la calidad del servicio brindado por la empresa a sus clientes. De acuerdo a los datos obtenidos por la presente investigación, los prestadores de servicios que atienden a la Comarca Lagunera reportan un área de oportunidad del $57 \%$ para mejorar su actuación.

El manejo adecuado de los flujos de bienes y servicios es de suma importancia, no solamente para reducir los costos asociados a los procesos de abastecimiento, producción y distribución, sino también para ofrecer una rápida respuesta a los requerimientos de los clientes. 


\section{Gráficas}

Gráfica 1. Problemas con los servicios logísticos.

\section{PROBLEMAS CON LOS SERVICIOS LOGÍSTICOS}

- Almacenamiento

- Transporte y distribución

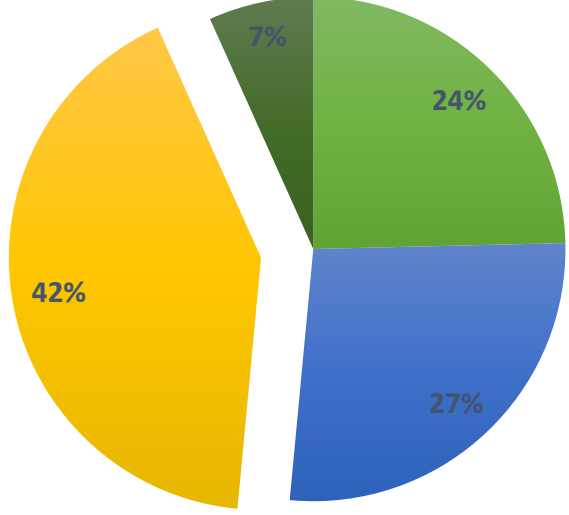

- Manejo de Inventarios

- Abastecimiento de Materia Prima

Fuente: elaboración propia.

Gráfica 2. Tipo de transporte más utilizado en exportaciones e importaciones.

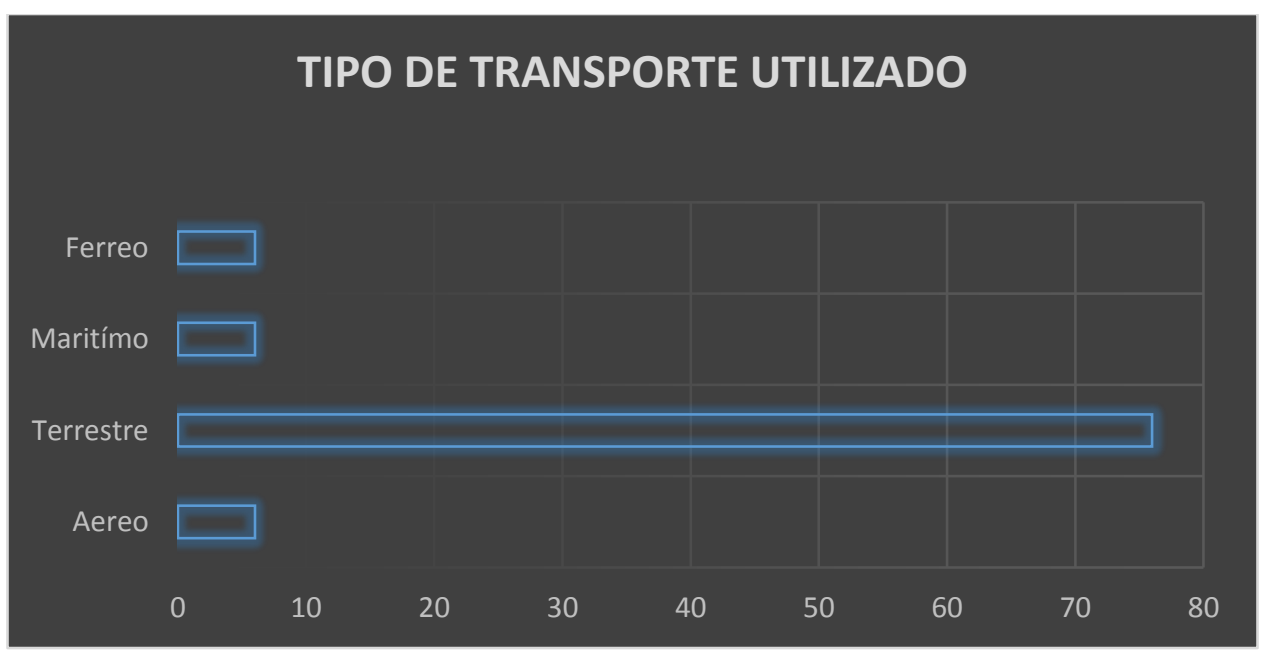

Fuente: elaboración propia. 
Gráfica 3. Evaluación de la calidad en el servicio logístico.

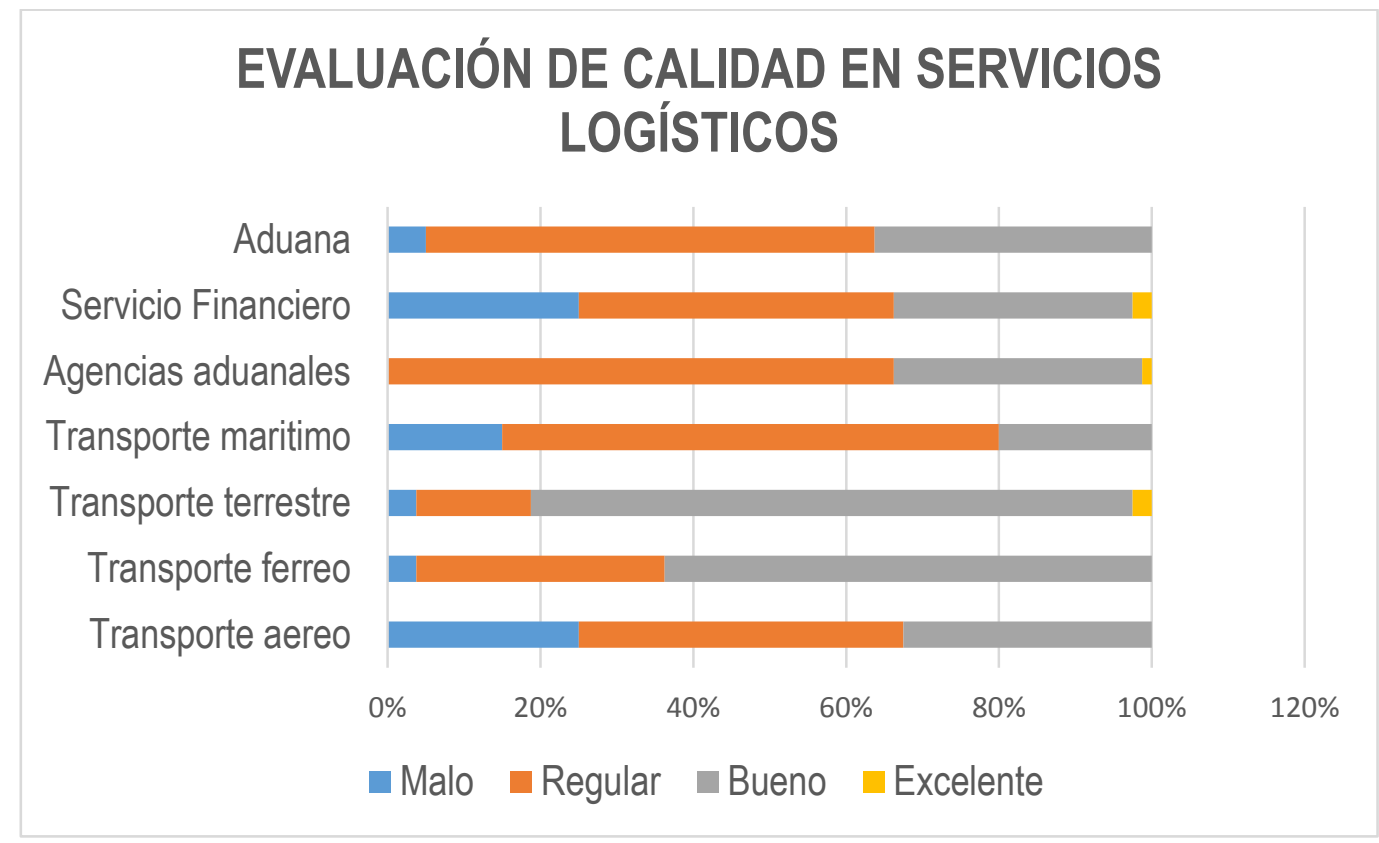

Fuente: elaboración propia.

Gráfica 4. Tiempos de atención en el servicio logístico.

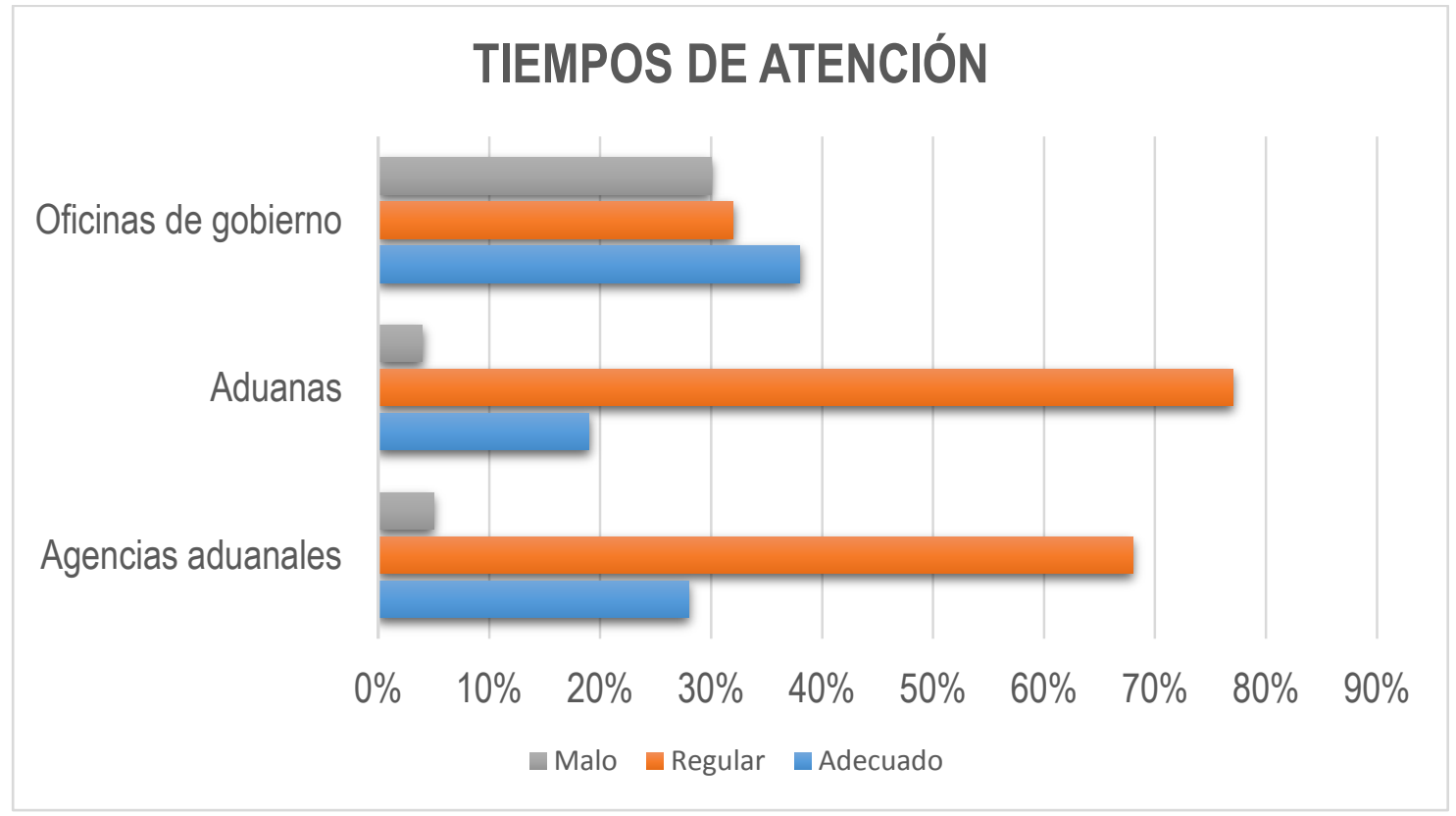

Fuente: elaboración propia. 


\section{Bibliografía}

A. Parasuraman, V. A. (20 de eNERO de 2017). American Marketing Association. Obtenido de http://www.jstor.org/stable/1251430

Aduanas, I. (. (2010). http://www.comercioyaduanas.com.mx/exportar. Obtenido de exportar: http://www.comercioyaduanas.com.mx/exportar

Banco de México. (12 de enero de 2017). Banxico educa. Obtenido de Banxico educa: http://www.banxico.org.mx/divulgacion/sistema-financiero/sistema-financiero.html

Cía Editora de la Laguna S.A. de C.V. (06 de enero de 2017). El Siglo de Torreón. Obtenido de El Siglo de Torreón: https://www.elsiglodetorreon.com.mx/noticia/263980.exporta-30-delas-empresas-laguneras.html

Cervantes, V.H. (2005). Interpretaciones del coeficiente alpha de Cronbach. Avances en Medición, 3, 9-28.

Concepto de definicion.es. (2014). conceptodedefinicion.es. Recuperado el 3 de octubre de 2016, de definicion de servicio: http://conceptodefinicion.de/servicio/

Confederación de Asociaciones de Agentes Aduanales de la República Mexicana. (12 de ENERO de 2017). CAAAREM. Obtenido de CAAAREM: http://www.caaarem.mx/web_caaarem/Resena.html

Delgado, J. H. (2011). Desarrollo de una cultura de calidad. México, D.F.: Mc.Graw Hill.

GESTIÓN DE LOS SERVICIOS SOCIO-SANITARIOS. (7 de Octubre de 2015). GESTIÓN DE LOS SERVICIOS SOCIO-SANITARIOS. Recuperado el 3 de Octubre de 2016, de GESTIÓN DE LOS SERVICIOS SOCIO-SANITARIOS.: http://www.aniortenic.net/apunt_gest_serv_sanit_4.htm

Grönroos, C. (1994). Marketing y estión de servicio:la gestión de los momentos de la verdad y la competencia en los servicios. Madrid: Díaz de Santos.

IDE-CESEM. (30 de ENERO de 2017). IDE-CESEM, Instituto de Directivos de Empresa, BUSINESS SCHOOL. Obtenido de IDE-CESEM, Instituto de Directivos de Empresa, 
BUSINESS SCHOOL: http://www.formacionparaprofesionales.es/servicio-clienteperspectiva-logistica/

INEGI. (6 de Enero de 2017). Instituto Nacional de Estadistica y Geografía. Obtenido de Instituto Nacional de Estadistica y Geografía: http://www.inegi.org.mx/geo/contenidos/geoestadistica/

INEGI. (6 de enero de 2017). Instituto Nacional de Estadística y Geografía. Obtenido de BOLETÍN DE PRENSA NÚM. 293/16 http://www.inegi.org.mx/saladeprensa/boletines/2016/balcom_o/balcom_o2016_07.pdf

Jhon Fredy Rave, J. C. (12 de enero de 2017). Academia Educativa. Obtenido de http://www.academia.edu/5364298/el_sena_BANCOS_DE_PRIMER_Y_SEGUNDO_PI SO

Julio Alvarez Botello, E. M. (12 de 01 de 2017). Enciclopedia Virtual "Eumed.net". Obtenido de Enciclopedia Virtual "Eumed.net": http://www.eumed.net/librosgratis/2014/1372/clientes-logisticos.html

Kotler, P. (2007). Marketing en el Sector Público. México, DF: Prentice-Hall.

Laura Fisher, A. N. (1994). Introducción a la investigación de mercado. México, D.F: Mc GrawHill Internacional S.A.de C:V.

Mercado Hernández, s. (2012). Comercio Internacional l. México, D.F: Editorial Limusa, S.A de C.V.

Milenio. (20 de Septiembre de 2014). Milenio.Com. Obtenido de Laguna: http://www.milenio.com/region/AgriculturaProtegida-MercadoExtranjeroComarcaLagunera_0_376162514.html

Paul R Murphy Jr, A. M. (2015). LOGISTICA CONTEMPORANEA. México, D.F: Person.

Principios de Gestion de Calidad. (2014). Sistemas de Gestion, Calidad, Medio Ambiente y PRL. Recuperado el 3 de Octubre de 2016, de Principios de Gestion de Calidad: http://abccalidad.blogspot.mx/2011/05/calidad-de-los-servicios.html 
PROCOLOMBIA. (25 de 1 de 2017). PROCOLOMBIA. Obtenido de PROCOLOMBIA: http://www.procolombia.co/node/162

Secretaría de Hacienda y Crédito Público. (05 de Enero de 2017). SAT Servicio de Administración Tributaría. Obtenido de http://www.sat.gob.mx/aduanas/importando_exportando/regimenes/Paginas/requisitos_pa ra_importar.aspx 